\title{
РАСПРОСТРАНЕННОСТЬ РАКА ЩИТОВИДНОЙ ЖЕЛЕЗЫ В НОВОСИБИРСКОЙ ОБЛАСТИ
}

\author{
'Шабельникова О.Ю., 'Бондарь И.А.
}

'ГБУЗ НСО «Государственная Новосибирская областная клиническая больница», Новосибирск ФГБОУ ВО «Новосибирский государственный медицинский университет» Минздрава России, Новосибирск

ЦЕЛЬ: изучить распространенность и заболеваемость раком щитовидной железы на территории Новосибирской области.

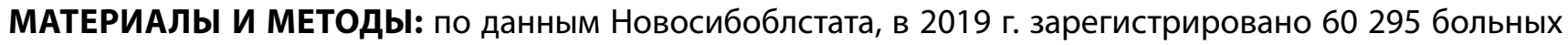
с патологией щитовидной железы, из них 23578 (39,1\%) имели узловую форму зоба. Рак щитовидной железы был у 970 человек, что составило 4,7\% от всех узловых форм зоба. Распространенность рака щитовидной железы в Новосибирской области в 2019 г. - 43,6 на 100 тыс. населения. Проведен ретроспективный анализ 970 амбулаторных карт пациентов, наблюдавшихся по поводу рака щитовидной железы. Данные представлены в виде среднего значения (M) и стандартного отклонения (SD), для сравнения частоты между группами использовали Х2.

РЕЗУЛЬТАТЫ И ИХ ОБСУЖДЕНИЕ: рак щитовидной железы встречался чаще У женщин 882 (90,9\%), средний возраст диагностики рака щитовидной железы был 50,0土14,8 года. Длительность наблюдения по данным амбулаторных карт варьировала от впервые выявленного до 46 лет, в среднем состави-

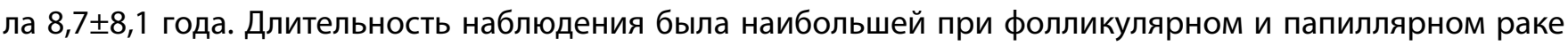

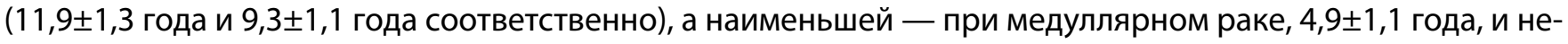
дифференцированном раке, 1,8土1,2 года. Все пациенты были прооперированы.

Наиболее распространенной формой рака был высокодифференцированный папиллярный рак у 79,6\% (n=772), реже встречались фолликулярный рак 16,8\% (n=163), медуллярный рак 3,0\% (n=29) и недифференцированный (анапластический рак) 0,6\% (n=6). Распространенность папиллярного рака щитовидной железы в Новосибирской области составила 34,7 на 100 тыс. населения, что достоверно выше по сравнению с фолликулярным раком - 7,3 на 100 тыс., медуллярным раком - 1,3 на 100 тыс. и недифференцированным - 0,27 на 100 тыс. населения. Лимфома щитовидной железы была у 1 пациента.

В 2019г.выявлено 66 новых случаев рака щитовиднойжелезы, заболеваемость составила 2,93 на 100 тыс. населения. Из них у 53 был папиллярный рак (2,4 на 100 тыс.), у 8 -фолликулярный рак (0,36 на 100 тыс.), у 5 - медуллярный рак (0,2 на 100 тыс.), новых случаев недифференцированного рака в 2019 г. не было. Возраст на момент диагностики варьировал от 10 до 85 лет, при папиллярном раке в среднем составил

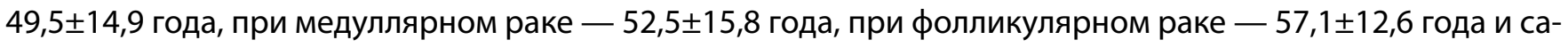

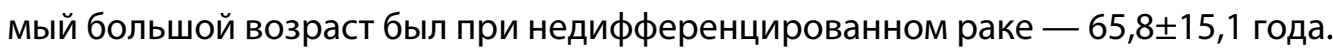

При папиллярном раке метастазы в региональные лимфатические узлы и легкие были у 15,5\% (n=120), радиойодтерапия проводилась в 16,7\% (n=129), у 2,5\% (n=19) потребовались повторные курсы радиойодтерапии в связи с прогрессированием рака, таргетную терапию сорафенибом получали 1,9\% (n=15). При фолликулярном раке радиойодтерапия проводилась в 9,8\% (n=16), у 1,2\% (n=2) были повторные курсы радиойодтерапии.

В 2019 г. умерли 0,6\% (n=6) больных, из них 3 - с папиллярным раком, 2 - с недифференцированным раком и 1 - с медуллярным раком.

ВывОДЫ: распространенность рака щитовидной железы на территории Новосибирской области составила 43,6 на 100 тыс. населения, заболеваемость - 2,93 на 100 тыс. Частота рака щитовидной железы была выше у женщин (90,1\%). Наиболее распространенной формой являлся папиллярный рак щитовидной железы (79,6\%). Частота проведения радиойодтерапии при высокодифференцированном раке составила 16,7\%, радиойодрезистентные формы были в 1,9\% случаев. Наибольшая длительность наблюдения была при фолликулярном раке щитовидной железы, а наименьшая - при недифференцированном раке. 